by McWeeney, who also includes nine of those mentioned by Bowles and Turner; one is briefly described by Bryant in the discussion on the latter paper ; while two additional ones are included in Mackenzie's tables. ${ }^{3}$ Summarising these cases we find that the accident occurs much more frequently in males, 24 patients being of that sex as compared with four females; in three the sex is not stated. The majority were of middle age, 17 being over 30 and nine below, while in five the age is not recorded. Alcohol seems to be a predisposing factor, since in 11 cases there was a history of drinking and two others were presumably alcoholic to a more or less degree. In nine a history of alcohol can be negatived, while in a similar number no mention of the habit is made. In all these cases the lesion was apparently brought about by the act of vomiting, though in two the exact cause is somewhat indefinitely stated. In three the vomiting was initiated by the impaction of food in the œsophagus, while in the case here recorded the ejection of the stomach contents was probably due to mechanical pressure on a loaded viscus as a result of the fall. In only one of the cases recorded has rupture of the oesophagus been the result of violence and in that instance it was only the indirect result, being brought about in a child by vomiting consequent on a fracture of the skull. In one of the records the situation and direction of the rupture are not stated, but in all the others it occurred in the lower third of the cesophagus, generally just above the diaphragm, and in some instances extending into the cardia, and in all except three was longitudinal in direction. In four the lesion was on the left side, in one on the anterior wall, while five were posterior, of which one was rather to the right and two were rather to the left. In 21 the aspect on which the lesion occurred is not given. The situation of the rupture in the lower third is probably accounted for by the fact that this portion of the cesophagus is covered only by involuntary muscle fibres, and experiments by Mackenzie, Bowles and Turner, and Brosch, ${ }^{4}$ who increased the pressure from within by means of either gases or fluids, proved that this was the weakest part, the rupture thus obtained being always longitudinal in direction and generally on the posterior surface. These facts give point to Turner's remark that in cases where a rupture can be diagnosed operative measures should be undertaken. Emphysema of the chest wall or of the neck and face was present in 16 cases and absent in three, while in 12 no mention is made of this phenomenon. Pneumothorax likewise was found in 16 instances, was absent in three, and not mentioned in 12, and was present in 11 of the cases in which emphysema occurred. All the cases were uniformly fatal, 22 dying within 24 hours. Two survived for as long as seven and a half days; in three the duration of life is not mentioned.

The symptoms accompanying the rupture were not directly observable in the case under notice, as the patient was in a comatose condition on admission to the hospital and a rupture of the cosophagus was not suspected during life. The etiology of such a rupture is a somewhat moot point. The chief views that have been put forward are those of Zenker and Ziemssen who attribute the possibility of such an accident to an intravital cesophago-malacia due to the regurgitation of acid and peptic matters from the stomach and retention of the same in the ceophagus with accompanying cessation or weakening of the circulation, and of MoWeeney who maintains that a softening of the wall occurs as the result partly of intravital digestion from retention of peptic matters and partly of inflammatory changes, as evidenced by his case in which the digestive process was accounted for by thrombosis of the vessels. Brosch ${ }^{5}$ as the result of his investigations states that a thinning of the walls may occur without any morbid lesion, though predisposing causes are to be found in such processes as the mechanical irritation of foreign bodies, ulceration, scarring, and endarteritis obliterans leading to necrosis. In the case here recorded there was no macroscopic evidence of any previous change and it seems possible that if such a lesion can be produced experimentally in the healthy osophagus by increasing the internal pressure such a result can be accounted for in the present instance by the great increase of abdominal pressure which is likely to have occurred in so severe an accident.

To Mr. Herbert Allingham my thanks are due for permission to make use of this case.

Mackenzie: Diseases of the Throat and Nose, vol. ii. 4 Broseh: Virchow's Archiv, Band clxii., 1900, p. 114. 5 Loc. cit.
The specimen will shortly be placed in the St. George's Hospital museum, No. 5331 [Series IX., 27A]. Grosvenor-street, $\mathbf{w}$.

\section{THE TREATMENT OF CANCER AND OTHER FORMS OF MALIGNANT DISEASE BY ELECTRIC} OSMOSE.

By CLARENCE A. J. WRIGHT, F.R.C.S. EDIN., F.F.P.S. GLASG., \&c.

THE increase of cancer and the interest that attaches to all measures tending towards its cure must be my apologg for bringing before the profession some experimental observations, crude and undigested though they be, on the results to be obtained in the treatment of the disease by electric osmose. In order that the method employed may be more easily understood I claim the indulgence of the reader for a very necessary digression, made to discuss some points bearing upon the subject. Among the more characteristic properties of high-frequency currents are those described as "the phenomena of electric osmose." To these it is necessary, in the first place, to allude.

The phenomena of electric osmose. - It has long been known that an electric current in passing through the liquid contained in a small vertical tube into which two platinum wires have been fused causes the level at which the liquid stands to be raised, when it moves in an upward direction. This phenomenon has been attributed to a mechanical current whose direction corresponds with that of the electric flux and whose velocity depends on its intensity, while the height to which the liquid can be raised is proportionate to the crosssection of the containing tube. As like effects are produced even when the tube is placed in a tank through which iced water is kept circulating, it is evident that the phenomenon can in no wise be said to depend upon the heat engendered by the passage of the current. Reinold and Rücker have noticed a somewhat similar phenomenon. When an upward current is passed through a perpendicular soap film it greatly retards, if it does not entirely arrest, the thinning of the film. Porrett observed that when a strong current was led into the liquid contained in a Daniel apparatus-a $U$-shaped container fitted with a porous diaphragm between the electrodes-a mechanical current was established which, by forcing a part of the fluid through the central partition, caused it to attain a higher level on the kathodic side of the diaphragm than on the anodic side. The degree to which this phenomenon, which is known as "electric endosmosis," is manifest depends upon the conductivity of the fluid and the specific dialytic ${ }^{\perp}$ capacity of the diaphragm for the liquid. With fluids that are bad conductors the phenomenon is more easily demonstrable than with those of good conductive capacity; at the same time it must be remembered that one that readily saturates the diaphragm is most readily passed through its pores. Closely allied to the preceding phenomenon is that known as "electric distillation." Although Baccaria had previously noticed that an electrified fluid evaporates more readily than an unelectrified one it was Gernez who first demonstrated this apparent distillation. He employed a bent glass-tube connecting two small glass globes into which the platinum electrodes had been fused. When liquid was introduced so that it stood at the same level in both globes and an electric current was passed through it, it was noticed that the fluid passed over from the anodic container to that connected with the kathode. This phenomenon cannot be attributed to any difference in temperature or extent of surface exposed, but rather to a slow creeping of the liquid along the inner surface of the connecting tube, which gives rise to a sort of electro-capillary syphonage. Quincke,

Reus explains the specific dialytic capacity of a diaphragm as the affinity it has for the liquid and the facilities it affords for transmission by capillary osmosis. For instance, linen has a greater dialytic capacity for water than either cotton, silk, or wool. Again, dialytic capacity for water than either cotton, silk, or wool. Again, when a capillary tube is introduced into different liquids it is noticed in the wh the liquid is at a higher level peripherally than centrally, as seen in dialytic affinity. 
on the other hand, discovered that an electric current is set up when a liquid is forced by pressure through a porous diaphragm. The electro-motive force of the current so induced is proportionate to the pressure and to the dialytic affinity of the diaphragm. "If Porrett's experiments," says Reus," be repeated with a fluid which is kept circulating through the porous diaphragm of a Daniel's apparatus by pressure (that in one arm being subjected to a positive pressure by means of an air pump, while a negative pressure is produced in the other by creating a partial vacuum) the rapidity with which the fluid passes through will be found to depend on the direction of the electric flux. It is increased when the direction of the electric flux and that of the current coincide and is diminished when they are opposed to each other. If instead of a simple fluid a standardised solution of two or more salts be employed it is found that the relative proportion of each in the dialysed fluid varies considerably from that of the standard solution, the amount present depending on (a) the specific diffusibility of the salt, $(b)$ the degree of its saturation in the standard solution, $(c)$ the nature of the diaphragm, and $(d)$ the duration of the electric flux. When two diaphragms are employed and the direction of the current periodically cataphoric treatment of disease by currents of high fre quency. In dealing with the phenomena of electric osmose attention was directed to certain methods by which an electric transference can be effected of substances dissolved in the liquid conveying the current through a series of diaphragms interposed between the electrodes, and the peculiar aptitude of alternating currents for effecting this was especially dwelt upon. An explanation of the method by which this result is produced is afforded us by the experiments of Tommasi and Thomas Tommasina. These show that the transmission of an electric impulse by an electrolytic conductor is accompanied by a transference of substance which manifests itself by the formation of arborescent chains of crystalline deposits between the electrodes. These chains follow the line of force of the electric field. Although it had long been known that the absorption of medicaments by the skin could be increased by electric action, ${ }^{2}$ yet it was not till the year 1890 that this method of medication attracted any real attention. The electro-chemical researches of Professor Foveau de Courmelles were the first attempt to treat the subject from a scientific standpoint. His investigations proved that cataphoresis is not simply a question of increased cutaneous absorption but of real

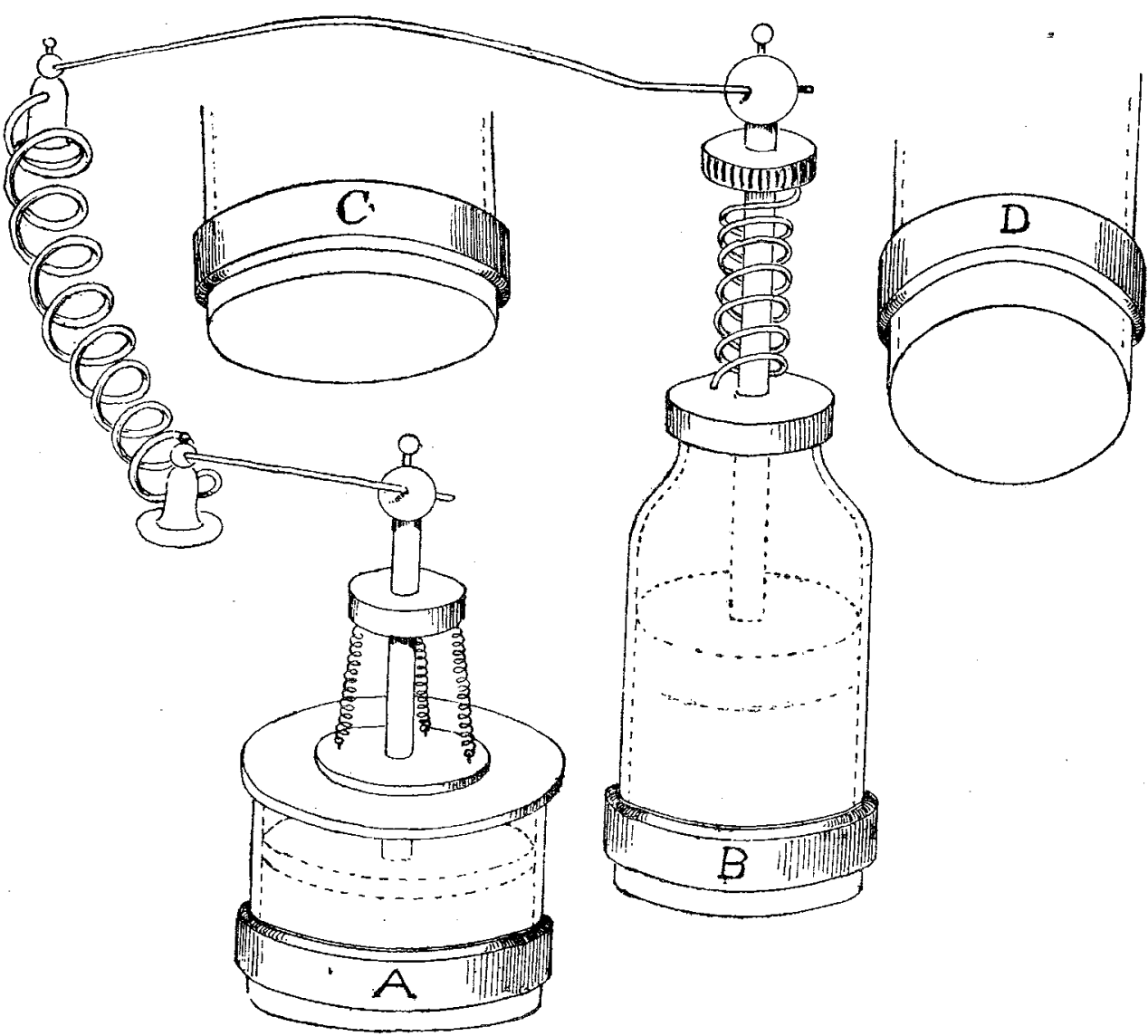

$A$ and $B$ show the catapboric cups attached to the opposite poles of the solenoid of high frequency. C and $\mathbf{D}$ show the diaphragms covering the bottom.

reversed the pressure of the liquid in the central compartment rises and causes the diaphragm to bulge outwards. Quantitative analysis of the fluids between and on either side of the diaphragms then shows that the percentage of the different salts present in the samples taken from each of the three compartments differ not only among themselves but also from that in the standard solution." This peculiar property of alternating currents is a point of great economical interest, as it affords a ready way of separating alkaloids and other active principles, both animal and vegetable. It also furnishes a clue to the method to be employed in obtaining gold from sea water, the question largely turning on the use of suitable diaphragms. More closely connected with electro-therapy is the explanation it affords of cataphoric phenomena and the movement of jons on the one hand and the resolution under electric treatment of tumours and chronic indurations on the other. It is these peculiar properties of alternating currents that are utilised in the cataphoric treatment of disease by high frequency.

Cataphoresis by high frequency. - Of all the methods employed in vibratory electrisation those classed under the head of "stabile bipolar derivation" are the best suited for penetration and particulate transference in accordance with certain fixed laws of the molecules of substance held in solution. These rass into the tissues which appear to act as a series of diaphragms interposed between the electrodes. The best known of these laws are coupled with the names of Faraday and Kohrasuch. According to Leduc the flux of force in electrolytes is made up of a double current of ions, an ascending current of anions passing towards the positive pole and a descending stream of kathions moving in the opposite direction. Leduc has also proved by means of soloured ions-a solution of permanganate of potash-that the medicaments do not merely pass into the subcutaneous areolar tissue, but directly penetrate into glandular organs and other structures more deeply situated. Frankenhaeusar has shown that the amount of medicament absorbed and the depth to which it penetrates have a constant relation, the former to the quantity, the latter to the intensity, of the current employed. He has also found that the anode is

2 This was first demonstrated in 1750 by Pivati, who employed a static charge for the purpose, and again in 1873 by Arthuis with electricity from the same source. In 1885 Lauret first utilised a continuous current in electric cataphoresis. 
instrumental in effecting an entrance into the tissues for acids, alkaloids and their salts, and metallic salts (other than those of the fixed alkalies and alkaline earths, which alone enter at the kathode). Oup electrodes are most commonly employed in cataphoresis, although the electric bath has also been used by Apostoli, Laquerriere, and Schnée. Phillips's method has been tried by Reus and found of some service when used in connexion with clay electrodes.

Having so far discussed the methods employed it will be interesting to note how much the reaction produced depends on the medicament introduced and the menstruum used to suspend it. The mineral acids cause a resolution of the skin and tissues, whilst the metallic salts give rise to necrosis. Water has a simple stimulating effect which varies with the intensity of the current employed. Alcohol gives rise to immediate coagulation of the albuminous constituents and causes a subsequent fibrosis. Liquid paraffin, without exerting any specific effect upon the tissues, allows of the ready transference to them of substances dissolved in it. Iodine, salicylate of soda, lithium salts, and pilocarpine produce their own specific reaction. Thus we find that iodine in both aqueous and alcoholic solutions is useful in givitre, tertiary syphilis, tabes and myelitis, and in effecting the resolution of secondary deposits in glandular organs. Salicylate of soda, oil of wintergreen, and Japanese peppermint are of service in arthritism resulting from traumatism, rheumatism, or specific disease; while lecithin, guaiacol, the glycero. phosphates, cinnamic acid, and the cacodylate of soda are beneficial in tuberculosis. It is evident from what has already been said in relation to Porrett's experiments that water, being a better conductor of electricity than either alcohol or liquid paraffin, is less likely to be of service in cataphoresis than the two last named. It requires, therefore, to be stiffened by admixture with either glycerine or alcohol before it is employed. Thus, in a cataphoric application of suprarenal extract as a preliminary ${ }^{3}$ to treatment by Finsen's light it was found that glycerine with normal salt solution proved a better menstruum for its suspension than water alone. In my experiments on cancer I employ either a dilute glycero-alcoholature or liquid paraffin emulsion as a vehicle for the salts employed. The chief of these are cinnamate of strontium, glycero-phosphate of lime and strontium, citric acid, iodine, and suprarenal extract. Cinnamate of strontium suspended in three parts of glycerine to five parts of distilled water is the medicament most frequently used and on which great reliance is placed to effect a cure. The glycero-phosphates have proved useful in assisting the casting off of a slough and promoting healing after an application of acid nitrate of bismuth. The citric acid was used for subduing neuralgic pains in the part, either alone or in conjunction with phenazonum. The use of lodine was chiefly confined to reducing the size of indurated glands and promoting absorption, although a strong solution was occasionally used for its escharotic effects on the tissues. It was then dissolved in pure alcohol. The suprarenal extract was employed as a preliminary to treatment at most of the sittings so as to limit absorption and to concentrate the action of the drug on the diseased tissues.

With these observations on the method of treatment pursued I will proceed to place before the reader the results obtained in five cases of cancer in which the treatment was beneficial.

CASE 1. Paget's disease.-A married woman, aged 51 years, the mother of seven children, the youngest of whom was 16 years, consulted me on March 19th, 1902, about a lump in her left breast which she first noticed six months previously when bathing. It was then of the size of half a walnut. As it was painless and caused her no inconvenience she took no notice of it till six weeks previously, when she consulted a medical man on account of a slight blood-stained discharge from the nipple. He pronounced the growth to be cancer and advised the removal of the breast. The general condition of the patient was that of a thin nervous woman, rather anæmic, and the subject of obstinate constipation. With the exception of a bilious attack at each menstrual period she had enjoyed good health till lately when she began to lose flesh. Menstruation commenced at 16 years of age and ceased at the age of 43 years. She had always suffered from cracked and tender nipples while nursing her children, but had not noticed any scaly patches on them till the discharge appeared. On examination a hard nodular mass of

3 It is found that when the tissues are rendered bloodless the effect of the light upon them is greatly increased. the size of a penny bun was found in the left mamma beneath the nipple which, being involved in the growth, was retracted and covered with a scaly crust. A similar eruption appeared on the areola which bled on its removal. Apart from this pressure on the mass caused a sanious discharge to exude from the nipple. The pigskin appearance of the overlying skin clearly showed that it was adherent to the subjacent tumour. It was impossible to lift or to roll the mass over the pectoral muscle. The axillary lymphatic glands were enlarged, two being of the size of a small walnut and others of about the size of a hazel-nut. The diagnosis of the case was simple and operation was advised. To this the patient was determined in no wise to submit. When cataphoresis by high frequency was suggested as an experiment she expressed $h \leftarrow r$ willingness to undergo the new method of treatment and was given a sitting of ten minutes' duration, three of which were with adrenalin solution ( 1 in 2500 ) and the remaining seven with a glycero-cinnamate of strontium. A dressing of lint soaked in weak infusion of cinnamon in which some citric acid had been dissolved was then applied to the breast and the patient was instructed to keep it constantly moist. On the 22nd the patient complained of having passed a restless night after the sitting. She was slightly feverish but slept better on the following day. On removing the dressing the nipple appeared raw but free from crust. Another sitting of the same duration and nature was given her. This treatment was continued until the middle of May, when on the 19th the ulceration around the nipple appeared to be quite healed and no discharge could be expressed from it. Though the lump was much smaller the skin was still very knobby and puckered. A sitting of three minutes' application of iodine was then made. This caused some pain and smarting at the time. On the two following days the breast was very swollen, red, and tender, so that six days were allowed to elapse before the next application was made. This being a weaker solution was better borne and gave rise to no irritation. From this time onwards the patient was treated to alternate applications of cinnamate of strontium and iodine till 46 sittings were made. On July 29th the growth had greatly diminished in size and consisted of seven separate nodules, each of the size of a hazel-nut, imbedded in a firm fibrous stroma. The skin, though still adherent, was more soft and supple. The enlarged axillary glands had quite disappeared. The patient had gained 18 pounds in weight during the preceding three months. Writing on Feb. 7th, 1903, she says: "The lumps appear, if anything, smaller and more freely moveable. You will be pleased to learn that I have gained another four pounds since last I saw you. I still use the citro-cinnamic dressings at bed-time and take my vigorine" (a mixture of calcium glycerophosphates with plasmon). The beneficial effects of cataphoric application of cinnamic acid are here well illustrated. Similar good results were obtained in Case 2 from its use.

OASE 2. Epithelioma of the bre st starting in the tubercles of Morgagni.- A single woman, aged 37 years, consulted me on May 17th, 1902, and gave the following history. "I was struck on the right breast three years ago by a cricket ball. This caused a deal of pain and discolouration which was more than a fortnight in disappearing. Early in November last I noticed a small pimple on the inner side of the nipple which I scratched. As it then festered I poulticed it to bring it to a head. The ulcer so caused has, in spite of treatment by caustics, salves, lotions, and scrapings, continued to spread. I have also some lumps under my arm which cause some pain in movement. I have been repeatedly advised to have my breast off, but dread the operation, as both my parents have died after operation for cancer (father, the tongue ; mother, rectum)." The patient was a flabby, florid-complexioned woman, in whom menstruation began at the age of 13 years and still continued. She had lived for years in the East, where she intended to return after her family affairs were settled. With the exception of malarial attacks and an attack of cholera she had enjoyed fair bealth. Her digestive tract was disordered by alcohol. Her liver was somewhat enlarged and so also was the spleen, but the kidneys were healthy, no sugar or albumin being found. On examination a large fungating ulcerative mass (three inches by two and a quarter inches) was seen covering the inner side of the nipple and spreading somewhat around it. The surface bled on the slightest touch. In a piece of tissue removed for microscopic examination typical cell nests were found. The growth had a deep attachment to the underlying tissues 
which were matted together. Several smaller hard nodular lumps were present in the outer half of the same breast. These involved the skin but were freely moveable over the underlying muscle. Both supraclavicular and axillary glands were enlarged ; some enlargement could also be felt beneath the great pectoral fold. Owing to the amount of pain felt in the part a citro-cinnamate dressing was ordered to be applied for three days before starting cataphoric treatment. On the 21st the patient was given a first sitting of five minutes' application of suprarenal extract and four minutes with eucalyptol-paraffin, after which the citrocinnamate dressing was again applied. The temperature rose the same night to $103 \cdot 6^{\circ} \mathrm{F}$. and gave rise to some hæmorrhage with much pain and discomfort. A mixture of citric acid and antipyrin was ordered. On the 22 nd she had passed a restless night but her temperature was now normal. The dressing was saturated with the discharge and its removal caused a reappearance of the hæmorrhage which, however, readily yielded to a spray of adrenalin solution (1 in 1000). Fresh dressings were applied and the arm was bandaged to the side. On the 24 th she had a second sitting of five minutes with suprarenal extract and ejght minutes with strong iodine glycero-alcoholature. Her temperature rose at night to $1026^{\circ}$ and was attended with more pain but no hæmorrhage. On the next day the wound was found to be sloughy and the parts around were much inflamed. The surface was dusted with xeroform and the citro-cinnamate dressings were reapplied. On the 25 th the wound continued to be very sloughy. A third sitting of three minutes with adrenalin and seven minutes with glycero phosphate solution was given. An iodo-eucalyptol dressing was applied. Pain had been great all day and the patient felt feverish with no appetite and great thirst. The evening temperature was $99.8^{\circ}$. The dressing was changed at bedtime for citro-cinnamate. On the 30 th the slough came away, leaving a clean, healthy surface. A fourth sitting of 10 minutes was given with adrenalin and cinnamate of strontium to the ulcer and an extra three minutes with weak iodine solution to the glands. The temperature at night was $100 \cdot 2^{\circ}$. On June $3: d$ fresh fungating granulations reappeared and the patient had a fifth sitting of 10 minutes with adrenalin solution for four minutes and acid nitrate of bismuth for six minutes. The glands were treated with iodine solution. This destroyed the fungating mass. On the 9 th the slough had come away and a sixth sitting of 10 minutes with adrenalin and glycerophosphate solution was given. On the 11 th a seventh sitting was given with adrenalin and cinnamate of soda. From this time the application consisted chiefly of cinnamate of strontium and the glycero-phosphates used at alternate sittings. On the $18 \mathrm{th}$ an axillary abscess was opened and the wound was swabbed out with iodo-eucalyptol solution. Towards evening cdema of the hands and arms appeared. The temperature rose to $103 \cdot 8^{\circ}$. The swelling of the arms took over a week to subside, although the temperature dropped to normal by the next morning. On August 14th the ulcer had almost completely cicatrised and much of the surrounding and underlying hardness had vanished. On the 19th the ulcer was perfectly healed and the swelling in the breast had almost entirely disappeared, very little being perceptible with the hand laid flat on the breast. The patient was now allowed to go to her friends. When next seen on Oot. 8th she was examined and the breast was found to have continued well. The skin over the ulcer was soft and paler. The swelling had all disappeared and she sailed for Singapore. Subsequent reports received all tell the same tale : "No recurrence; no swelling; no pain. Nothing but the scar to remind me of the previous trouble." Microscopic examination proved this to have been a true epithelioma which appeared to have started in the tubercles of Montgomery and to have spread most towards the sternum. I cannot recall any case having a similar origin. The swabbing-out of the abscess cavity with strong iodoencalyptol solution was undoubtedly the cause of the cdema. The great efficacy of bismuth salts in getting rid of the recurrent fungoid growth is to be remarked.

CASE 3. Malignant ulceration of the vaginal cervix.A married woman, aged 32 years, the mother of two children, came to me on May 3rd, 1902, complaining of a foul vaginal discharge attended by hæmorrhage after intercourse. She complained also of dyspeptic symptoms (reflex?) and inability to indulge in much outdoor exercise. Her family history was good and her general health, with the exception of constipation and dyspeptic trouble, was fair. Examination per vaginam showed some ulcerative excavation of the vaginal cervix with enlargement of the lymphatics. The uterus was fairly moveable but heavy. Scraping from the surface confirmed the diagnosis of cancer. This case was treated by effluvation with Bisserie's electrode and cataphoresis by high frequency. The applications employed were chiefly cinnamate of soda and dilute solution of iodine. After each sitting the vagina was tamponed with a glycerolate of citro-cinnamic acid. After 12 sittings the ulcer ceased to spread and took on a healthy action. Seven subsequent sittings completed the treatment. There has been so far no recurrence of the disease, either locally or in other organs. The constipation, which was a marked symptom in the case, readily yielded to treatment by the method devised by Marie of Toulouse.

CASE 4.-Recurrence after extensive operation.-The patient was a man, aged 73 years, from whose breast a malignant growth had been removed 14 months previously, and who consulted me on August 14th, 1902, for two recurrent nodules in the scar of operation. 12 sittings with iodine and cinnamate of strontium sufficed for their entire absorption. A recurrence in the axillary wound in November of the same year yielded to treatment after seven sittings.

CASE 5. - Melanotic cancer starting in a pigmented mole on the tarso-metatarsal joint of the great toe. - This patient, a man, aged 45 years, consulted me on May 23rd, 1902, about "an inflamed bunion" on the great toe of his right foot. He had from birth had a pigmented mole on the inner side of the tarso-metatarsal articulation, which became inflamed some nine months previously and had continued in much the same condition ever since. He had now, in addition, a nodular mass in his groin, the size of a goose's egg. Examination disclosed a melanotic cancer of the part with secondary induration of the inguinal glands. The part was treated with acid nitrate of bismuth, cinnamic acid, and iodine, and he was discharged cured on Oct. 27th. In December last he showed me several flesh moles that had since come out and taken on a similar action. These were small and yielded to treatment in from seven to 18 sittings. His last letter from the United States, dated April 21st, 1903 , declared him to be still free from any further manifestation of the disease.

It must not, however, for an instant be supposed that I have met with similar success in all cases that have under. gone this method of treatment. Of the 23 cases of which I have notes, in the five above-mentioned it proved successful. In two others it signally falled either to arrest the disease or to prevent secondary growth in the internal organs. Both these cases were, however, from the first hopeless in the extreme, both from the degree of cachexia present and from the disease having already spread to the lymphatics of the trunk. In 16 cases treatment was abandoned after sittings varying from four to ten in number, some preferring to undergo operation and others treatment by the $x$ rays and Finsen's light. Yet if all these be accounted failures I would venture to suggest that 22 per cent. of successes is still sufficiently encouraging to recommend this method of treatment to the attention of the profession for a more extended trial than $I$ have yet given it. It is not put forward as a cure for cancer but as a suggestion of the line of treatment to be adopted till that is really discovered. I personally believe that a "radio-active salt of strontium, introduced into the tissues by cataphoresis by high frequency," will prove the desired panacea of all forms of malignant disease. With these suggestions I leave the pursuit to others better fitted to carry it to a successful issue.

Leytonstone.

\section{THE TREATMENT OF PLAGUE BY LARGE DOSES OF CARBOLIC ACID GIVEN INTERNALLY.}

By J. MITFORD ATKINSON, M.B. LOND., M.R.C.S. FNG., PRINCIPAL CIVIL MEDICAL OFFICER, HONG-KONG.

IN 1899 I published in THE LANCET ${ }^{1}$ the notes of a case of bubonic plague treated successfully by large doses of carbolic acid. I was led to try this remedy from reading an article on the treatment of influenza in a similar way. The patient above referred to was a Scotchman and he came into hospital practically as soon as he was attacked by the

1 The Lancet, Dec. 9th, 1899, p. 1589. 